Document downloaded from:

http://hdl.handle.net/10251/102249

This paper must be cited as:

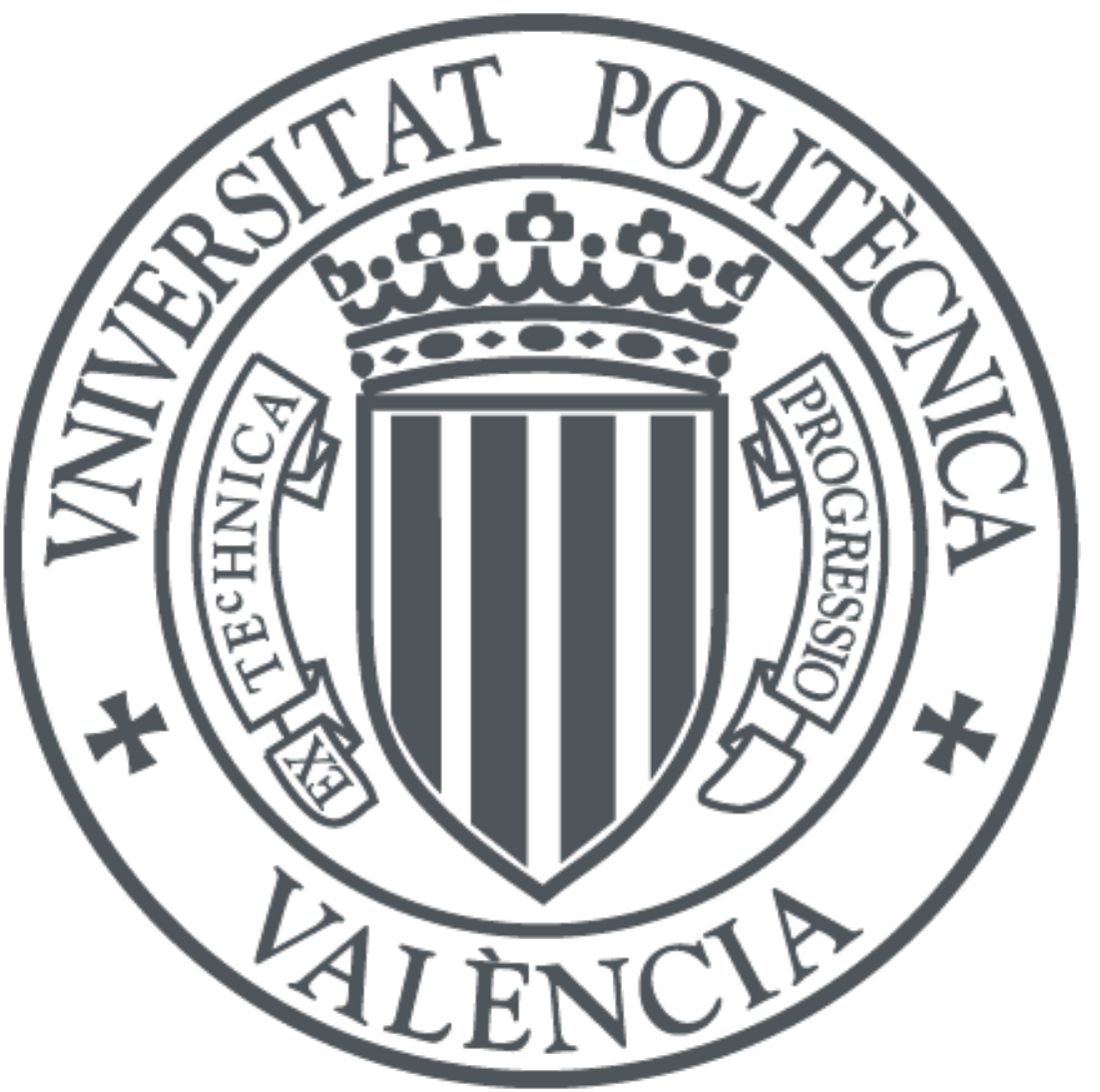

The final publication is available at

http://doi.org/10.1109/LAWP.2016.2614342

Copyright Institute of Electrical and Electronics Engineers

Additional Information 


\title{
Microwave Planar Lens Antenna Designed with a Three-Layer Frequency Selective Surface
}

\author{
Daniel Sánchez-Escuderos, Member, IEEE, Hon Ching Moy-Li, Eva Antonino-Daviu, Member, IEEE, \\ Marta Cabedo-Fabrés, Member, IEEE, Miguel Ferrando-Bataller, Member, IEEE,
}

\begin{abstract}
A multilevel Frequency Selective Surface (FSS) formed by a non-uniform distribution of circular holes on a metallic sheet is presented. The FSS is aimed at working as a planar lens to increase the gain of the feeding aperture. The unit cell of the FSS acts as a high-pass filter that provides the necessary phase shift to focus the beam on the far-field region. The phase shift is synthesized by suitably configuring the size and position of each unit cell in the FSS. The planar lens is placed next to the feeding aperture to confer the structure a low-profile shape. A prototype has been designed, fabricated and measured. Results show an increment of $7.32 \mathrm{~dB}$ in the gain compared to the feeding aperture within a $7.33 \%$ bandwidth around $20.45 \mathrm{GHz}$, and a side-lobe level below $-12 \mathrm{~dB}$, with an aperture efficiency of $65 \%$ and a radiation efficiency above $90 \%$.
\end{abstract}

Index Terms-Frequency selective surfaces (FSS), lens antennas, reflector antenna feeds, satellite antennas.

\section{INTRODUCTION}

$\mathbf{M}$ ULTIPLE-BEAM antennas have experienced a considerable growth in Ka-band satellite communications during last years. The satellite capacity in this kind of architectures is considerably increased with respect to the traditional contoured coverages due to the re-use frequency scheme and the increased gain of spots [1]. In order to provide multiplebeam coverage, a large reflector is commonly employed while the feeder is physically shifted to point the radiation pattern towards the desired spot position on Earth [1]. Contiguous spot beams require adjacent feeds but, since a high system performance requires a directive horn, feeds may overlap [1]. Consequently, other solutions must be envisaged.

A four-reflectors configuration is the most commonly adopted solution, e.g. the Ka-band Eutelsat system [2]. However, the high weight and cost of these systems have led to propose alternative solutions that use only two reflectors. These solutions implement a multiple-feed per beam configuration by exciting several apertures at the same time at the expense of increasing the complexity of the feeding network [3].

Another solution implements a single-feed per beam architecture by using a Frequency Selective Surface (FSS) in front of an array of apertures. FSS's are periodic structures capable of improving the performance of an antenna at certain

This work was supported by the Spanish Ministry of Economics and competitiveness under project TEC2013-47360-C3-3-P, and by Generalitat Valenciana under project GV/2015/065.

D. Sánchez-Escuderos, H. C. Moy-Li, E. Antonino-Daviu, M. CabedoFabrés and M. Ferrando-Bataller are with the Instituto de Telecomunicaciones y Aplicaciones Multimedia (ITEAM) of the Universitat Politècnica de València, c/ Cami de Vera s/n, 46022 Valencia, Spain (e-mail: dasanes1@iteam.upv.es, chingmoy1@gmail.com, evanda@dcom.upv.es, marcafab@dcom.upv.es, mferrand@dcom.upv.es)

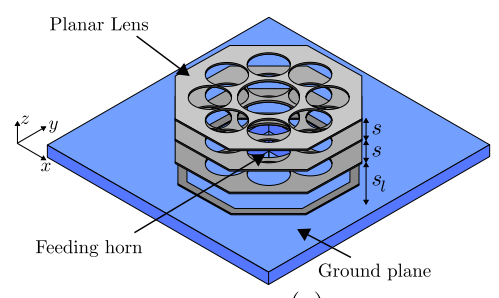

(a)

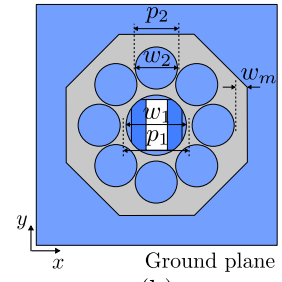

(b)
Fig. 1. Three-layer non-uniform FSS working as a planar lens in front of a square aperture: (a) 3D view, and (b) upper view.

frequency bands or polarizations with a small increment of the structure's profile [4].

A simple FSS is the so-called metallic hole array, whose unit cell is a perforated metallic sheet. This kind of FSS has been recently used to enhance the directivity of a circular aperture in the X-band [5], or to design a multiple-beam antenna in the Ka-band [6]. These proposals present good performance, but they suffer from narrow bandwidth, caused by the use of electrically-small unit cells to form the FSS.

In this letter, the three-layer non-uniform FSS shown in Fig. 1, based on the circular-hole unit cell described in [7], is proposed. The FSS is aimed at working as a planar lens to increase the directivity of the feeding aperture by suitably compensating the phase of the incoming waves from the feeder. The bandwidth of the resulting planar lens is considerably increased with respect to previously proposed solutions by using large unit cells. An equivalent circuit model for the proposed unit cell that accounts for the large size of the unit cell is presented. A prototype has been fabricated and measured for validation.

\section{UNIT CELL: EQUIVALENT CIRCUIT}

The unit cell of the proposed non-uniform FSS is a perforated metallic sheet with period $p$ and diameter of the central hole $w$ (see Fig. 2 (a)). Typically, the period of this kind of unit cell is below $\lambda / 2$ [8]. However, in this letter, a larger period is used to increase the bandwidth of the unit cell.

Additionally, the number of layers determines the maximum phase shift that the unit cell can produce. Considering an optimized gap between layers $(s)$ of $4.75 \mathrm{~mm}$, i.e., $\lambda / 3$ at $20.45 \mathrm{GHz}$, a three-layer unit cell (shown in Fig. 2 (b)) can provide up to $200^{\circ}$ of phase shift, as demonstrated in [7].

Fig. 2 (c) illustrates the equivalent circuit of the three-layer unit cell. As can be observed, each layer is formed by a series capacitor and inductance, connected in parallel to an 


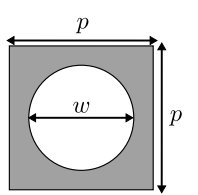

(a)

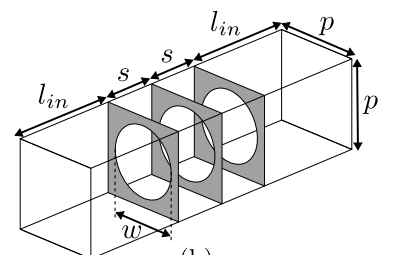

(b)

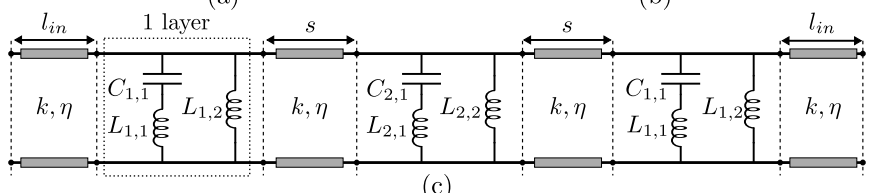

(c)

Fig. 2. Perforated metallic sheet: (a) circular hole unit cell, (b) three-layer unit cell, and (c) equivalent circuit.

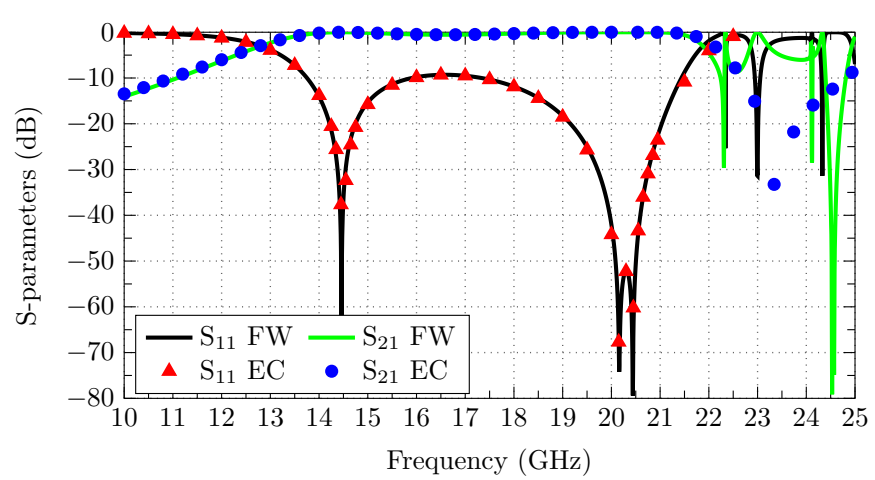

Fig. 3. S-parameters of the full-wave (FW) simulation and its equivalent circuit (EC) of a three-layer unit cell with $s=4.75 \mathrm{~mm}, p=13.5 \mathrm{~mm}(0.89 \lambda$ at $20.45 \mathrm{GHz}$ ) and $w=12.15 \mathrm{~mm}$.

inductance. Note that this equivalent circuit is different from the conventional equivalent circuit for an inductive grid [8] since the grid's period is larger than $\lambda / 2$. Fig. 3 shows a comparison between the full-wave simulation of a three-layer unit cell, and its equivalent circuit (Fig. 2 (c)).

The values of the lumped elements in the equivalent circuit have been determined with AWR [9], giving $C_{1,1}=8.65 \mathrm{fF}$, $C_{2,1}=12.82 \mathrm{fF}, L_{1,1}=4.573 \mathrm{nH}, L_{1,2}=2.836 \mathrm{nH}, L_{2,1}=2.148 \mathrm{nH}$ and $L_{2,2}=2.975 \mathrm{nH}$. These values present a linear behavior with respect to the period, as shown in Fig. 4 (note that, despite results are provided for $w=0.9 p$ to get a large bandwidth, the same trend is observed for other diameters). Therefore, the equivalent circuit for any period can be easily known, first, calculating the lumped elements for two different periods, and, then, applying a linear regression to the obtained values.

The use of a large hole diameter $w$ provides a large bandwidth, which, in turn, permits the use of the layered scattered approach [10]. This technique is based on shifting the frequency response of a filter so that, within a certain frequency range, the amplitude of the transmission parameter is kept constant, whereas its phase is changed.

In order to illustrate this technique, Fig. 5 shows the transmission parameter of a three-layer unit cell with several periods. As can be observed, from $19 \mathrm{GHz}$ to $22.7 \mathrm{GHz}$, the amplitude of the transmission parameter is kept above $-2 \mathrm{~dB}$ for all periods whereas the phase is shifted. Within the frequency range of interest, $19.7 \mathrm{GHz}-21.2 \mathrm{GHz}$ (see

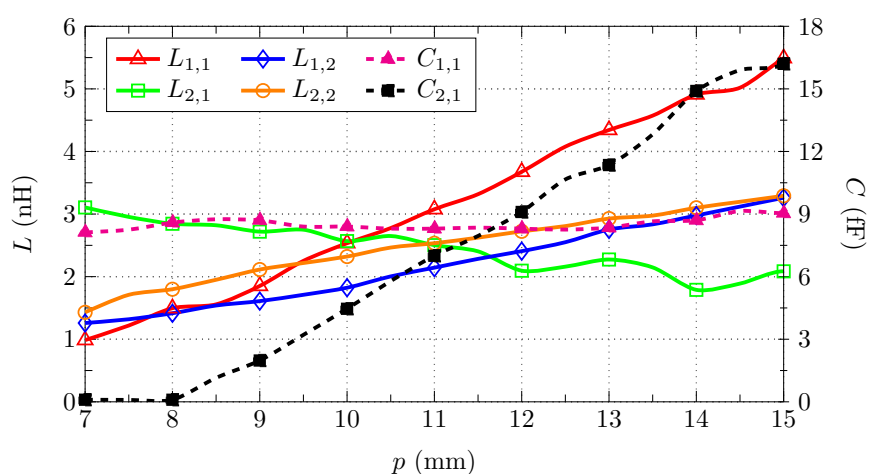

Fig. 4. Lumped elements values (LC) vs unit cell period $p$ with $w=0.9 p$.

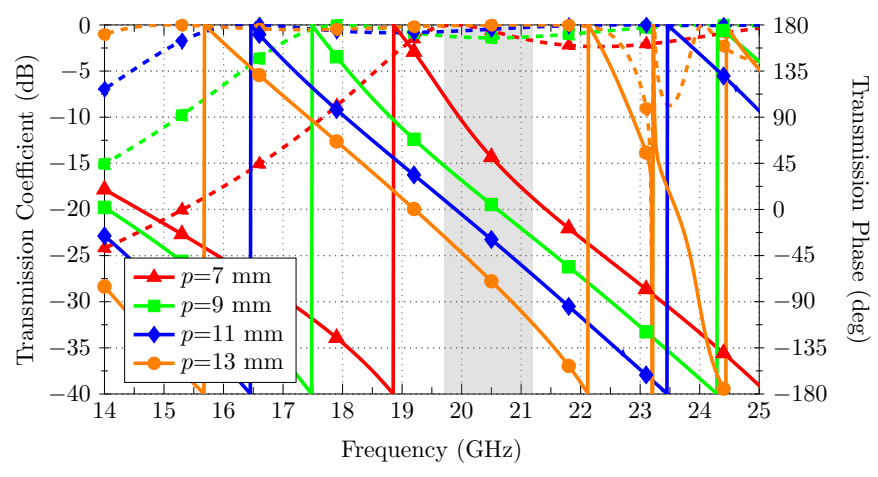

Fig. 5. Absolute value (dashed lines) and phase (solid lines) of the transmission parameter of a three-layer unit cell with different periods $p_{i}$ $\left(w_{i}=0.9 p_{i}\right)$ and $s=4.75 \mathrm{~mm}$.

shadowed area in Fig. 5), a phase shift of up to $137^{\circ}$ can be obtained. A larger phase shift might be reached by increasing the range of periods, or incrementing the number of layers [7].

\section{MICROWAVE PLANAR LENS}

Microwave planar lenses are typically formed by receiving and transmitting antennas connected through a phase delay mechanism [10]. The different elements are designed to achieve a certain field distribution on the transmitting face of the lens. This letter proposes a planar lens aimed at obtaining a uniform amplitude and phase field distribution on the upper level of the lens to focus the beam on the far-field region [11].

A sketch of the proposed planar lens is depicted in Fig. 6. As it can be seen, the planar lens is illuminated by a square aperture with a spherical phase distribution. The size of the square aperture, obtained by widening a standard rectangular waveguide (WR-42) in the XZ plane, is $10.668 \times 10.668 \mathrm{~mm}$.

The proposed planar lens, shown in Fig. 1, must compensate the phase shift caused by the different path length $(\Delta S=$ $S_{2}-S_{1}$ ) of the incoming waves in order to produce a uniform phase distribution on the upper level of the lens. The maximum path length, determined by the extension of the lens and the distance between the lens and the feeding aperture $\left(s_{l}\right)$, limits the maximum available bandwidth, as illustrated in Fig. 5.

Considering the phase shift produced by the different periods (see Fig. 5), a relationship between $s_{l}$ and the operating bandwidth can be deduced. Fig. 7(a) shows graphically this 


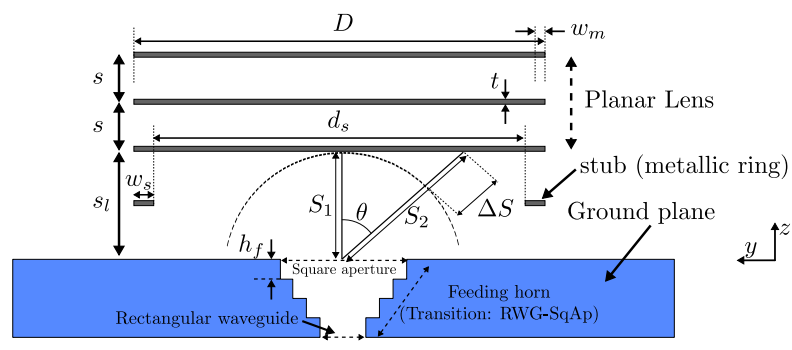

Fig. 6. Lateral view of the three-layer FSS working as a planar lens in front of a square aperture.

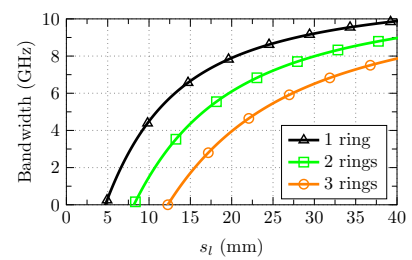

(a)

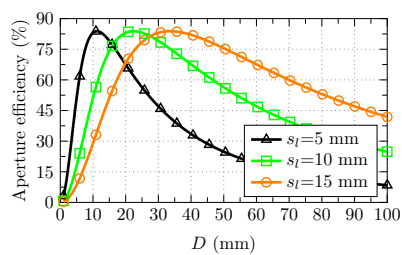

(b)
Fig. 7. Electrical parameters vs geometrical dimensions: (a) Bandwidth vs lens separation for a three-layer configuration and a central frequency of $f_{0}=20.45 \mathrm{GHz}$, and (b) Aperture efficiency vs lens diameter $(D)$.

relationship for several number of rings around the central element (this letter only considers one ring, see Fig. 1). As it can be seen, the higher $s_{l}$ is, the larger the bandwidth is.

Nevertheless, the choice of $s_{l}$ must also consider the aperture efficiency of the lens $\left(\eta_{a p}\right)$, which can be split into the illumination efficiency $\left(\eta_{i l}\right)$ and the spill-over efficiency $\left(\eta_{s}\right)$ as: $\eta_{a p}=\eta_{i l} \eta_{s}$. If the area of the lens is approximated by a circle of diameter $D$ (see Fig. 6), and the field radiated by the feed is assumed to be of type $\cos (\theta)^{2.5}$ for $z<20 \mathrm{~mm}$, the following formulas for $\eta_{i l}$ and $\eta_{s}$ can be deduced [12]:

$$
\begin{gathered}
\eta_{i l}=\frac{D^{2}(n-1)}{4(n-2)^{2}} \frac{\left(4 s_{l}^{2}-\left(1+\frac{D^{2}}{4 s_{l}^{2}}\right)^{-n / 2}\left(D^{2}+4 s_{l}^{2}\right)\right)^{2}}{4 s_{l}^{2}-\left(1+\frac{D^{2}}{4 s_{l}^{2}}\right)^{-n}\left(D^{2}+4 s_{l}^{2}\right)} \\
\eta_{s}=1-\cos \left(\arctan \left(D /\left(2 s_{l}\right)\right)\right)
\end{gathered}
$$

Fig. 7(b) shows the aperture efficiency, calculated from (1) and (2). This figure indicates that for a diameter above $30 \mathrm{~mm}$, the larger $s_{l}$ is, the higher the aperture efficiency results, which is mainly caused by the good illumination efficiency.

The above results illustrate that a very small separation $s_{l}$ (to minimize the profile) may degrade the response of the lens. In this letter, a separation $s_{l}=9.5 \mathrm{~mm}$ has been chosen as a tradeoff between low profile, bandwidth and aperture efficiency. This separation corresponds to a phase compensation between the central element and the surrounding elements of around $94^{\circ}$, as shown in Fig. 8 (note that the center of the surrounding elements is located at, approximately, $y= \pm 12 \mathrm{~mm}$ ).

Additionally, a metallic ring is inserted at an intermediate level between the lens and the feeding aperture (see Fig. 6). This ring works as a stub that minimizes the leakage in the air gap between the lens and the square aperture.

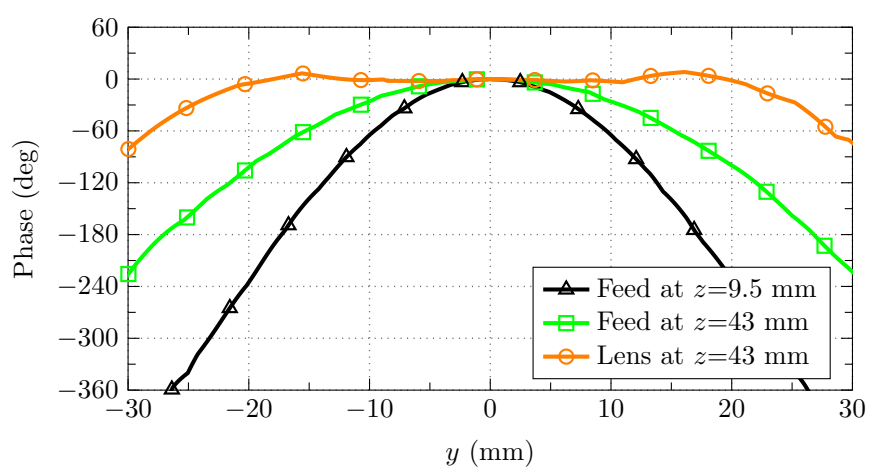

Fig. 8. Phase of the $y$ component of the Electric field with (marked as Lens) and without (marked as Feed) lens at several levels on the YZ plane.

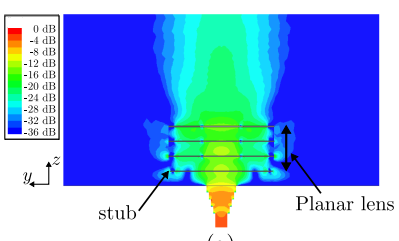

(a)

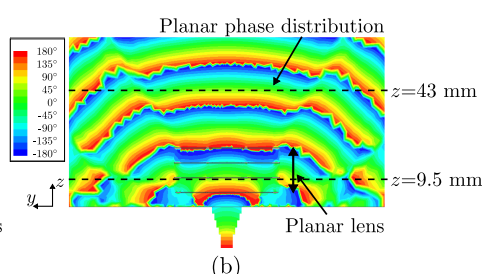

(b)
Fig. 9. $y$ component of the electric field $\left(E_{y}\right)$ on a vertical/transverse plane (YZ) at $20.45 \mathrm{GHz}$ : (a) absolute value, and (b) phase.

\section{PRototype AND MEASUREMENTs}

The proposed microwave planar lens has been designed to operate in the range 19.7-21.2 GHz. By examining the phase of the transmission parameter of the unit cell shown in Fig. 5, an initial estimation for the size of holes (see Fig. 1(b)) can be established in $p_{1}=13 \mathrm{~mm}, p_{2}=8 \mathrm{~mm}, w_{1}=11.7 \mathrm{~mm}$ and $w_{2}=7.2 \mathrm{~mm}$. Note that these values correspond to an infinitelyperiodic and uniformly-distributed unit cell but, in Fig. 1, cells are arranged along a circle. In order to correct this difference, an optimization becomes mandatory. To this aim, the period of the cells, and the size of the stub, have been optimized by a parametric study to maximize the directivity at broadside and minimize the side-lobe level. The optimized dimensions with $s=4.75 \mathrm{~mm}, w_{m}=2 \mathrm{~mm}$ and $t=350 \mu \mathrm{m}$ are: $p_{1}=14.18 \mathrm{~mm}$, $p_{2}=9.98 \mathrm{~mm}, w_{1}=12.76 \mathrm{~mm}, w_{2}=8.98 \mathrm{~mm}, d_{s}=33.25 \mathrm{~mm}$, $w_{s}=1.94 \mathrm{~mm}$ and $h_{f}=2.5 \mathrm{~mm}$, which means $D=38.14 \mathrm{~mm}$.

Fig. 9 shows the $y$-component of the electric field on the $\mathrm{YZ}$ plane of the lens. As it can be seen, the absolute value is constant on the upper plane of the lens (see Fig. 9(a)), and the phase is transformed from a spherical distribution in the gap between the feeding aperture and the lens (see Fig. 8) into a uniform distribution above the lens (see Fig. 9(b) and Fig. 8).

The optimized planar lens has been manufactured for validation purposes. Fig. 10 depicts three pictures of the prototype, formed by three copper layers, a metallic ring working as a stub and a foam frame to guarantee the air gap between layers.

The $S_{11}$ parameter of the microwave planar lens, plotted in Fig. 11, shows that the proposed structure is well matched $\left(\mathrm{S}_{11}<-15 \mathrm{~dB}\right)$ within the frequency range of interest.

The radiation pattern of the microwave planar lens has been measured with and without stub at several frequencies. Results are compared in Fig. 12 to the measured radiation 


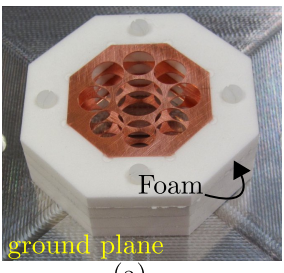

(a)

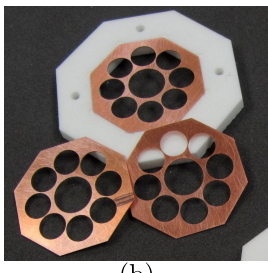

(b)

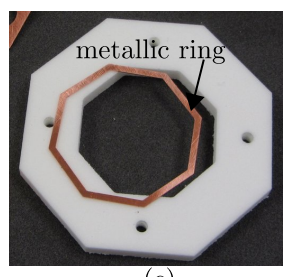

(c)
Fig. 10. Prototype of the proposed microwave planar lens: (a) complete structure, (b) separated layers, and (c) intermediate stub.

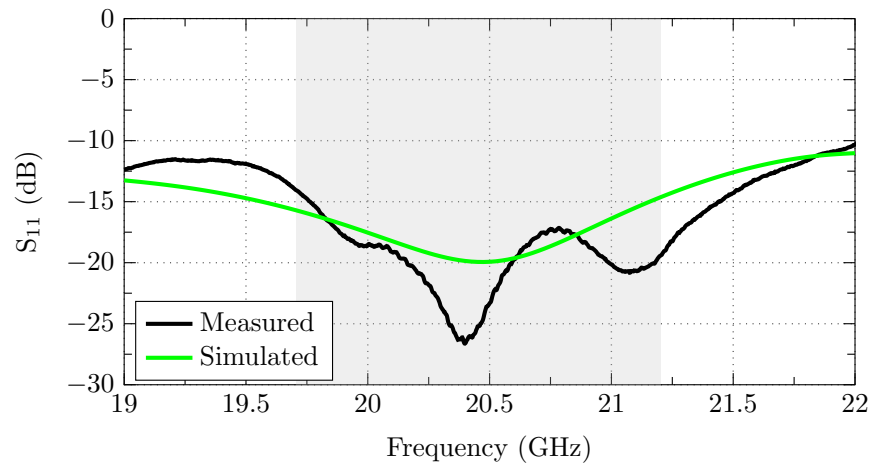

Fig. 11. Measured and simulated $\mathrm{S}_{11}$ parameter of the fabricated microwave planar lens (shadowed area: 19.7-21.2 GHz)

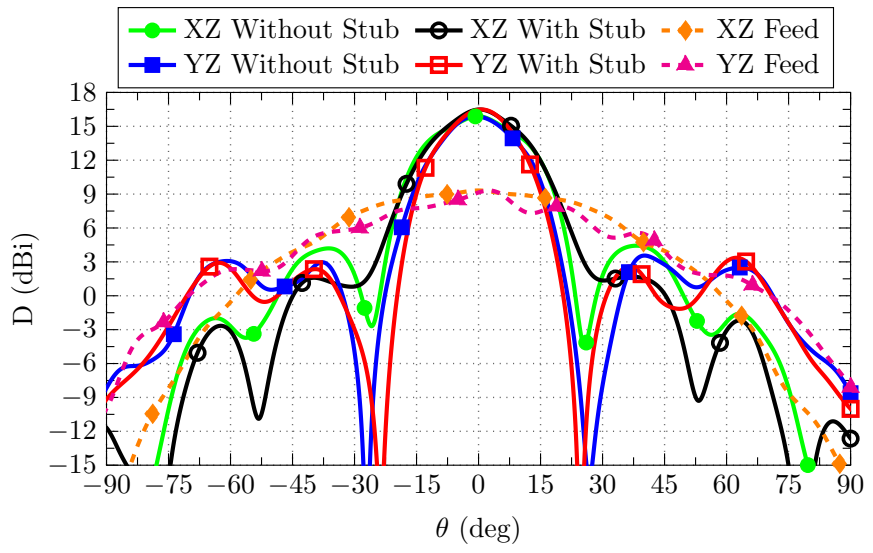

Fig. 12. Measured directivity of the microwave planar lens with and without stub (solid lines) and the isolated feeding aperture (dashed lines) on the $\mathrm{XZ}$ plane (H-plane) and the YZ plane (E-plane) at $20.45 \mathrm{GHz}$.

pattern of the isolated feeding aperture. As can be observed, the maximum directivity in the planar lens is increased $7.32 \mathrm{~dB}$ with respect to the maximum directivity of the isolated feeding aperture. The side-lobe level is $-11.36 \mathrm{~dB}$ without stub and $-13.69 \mathrm{~dB}$ with stub, what means an increment of $2.33 \mathrm{~dB}$ in the solution with stub due to the reduction of the lateral leakage. The average aperture efficiency of the planar lens within the frequency range of interest is $65 \%$, which matches with the value predicted theoretically in Fig. 7(b).

Fig. 13 shows the measured maximum gain and the radiation efficiency of the proposed structure. As can be observed, the maximum gain is above $17 \mathrm{dBi}$ at all frequencies and the radiation efficiency is above $90 \%$. This high efficiency is caused by the use of low-loss materials in the prototype.

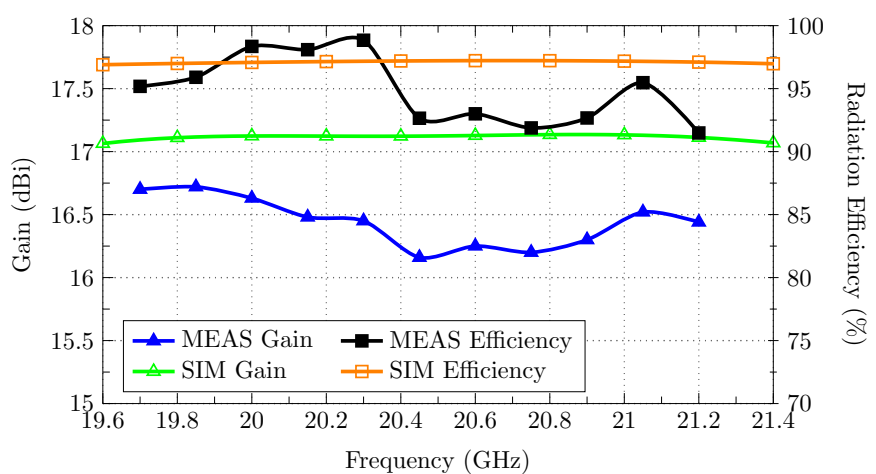

Fig. 13. Gain and radiation efficiency of the fabricated planar lens.

\section{CONCLUSION}

This letter presents a three-layer FSS working as a microwave planar lens from $19.7 \mathrm{GHz}$ to $21.2 \mathrm{GHz}$. A metallic ring is inserted in the air gap between the lens and the feeding aperture to minimize the lateral leakage. Measurements of the manufactured prototype show a good return loss level, and a uniform maximum directivity and side-lobe level within the operating frequency range. The use of low-loss materials provides a radiation efficiency above $90 \%$, whereas the good illumination of the lens gives an aperture efficiency of $65 \%$.

\section{REFERENCES}

[1] S. K. Rao, "Advanced antenna technologies for satellite communications payloads," IEEE Transactions on Antennas and Propagation, vol. 63, no. 4, pp. 1205-1217, 2015.

[2] H. Fenech, S. Amos, A. Tomatis, and V. Soumpholphakdy, "High throughput satellite systems: An analytical approach," IEEE Transactions on Aerospace and Electronic Systems, vol. 51, no. 1, pp. 192-202, 2015.

[3] J. M. Montero, A. M. Ocampo, and N. J. Fonseca, "C-band multiple beam antennas for communication satellites," IEEE Transactions on Antennas and Propagation, vol. 63, no. 4, pp. 1263-1275, 2015.

[4] Y. E. Erdemli, K. Sertel, R. Gilbert, D. E. Wright, and J. L. Volakis, "Frequency-selective surfaces to enhance performance of broad-band reconfigurable arrays," IEEE Transactions on Antennas and Propagation, vol. 50, no. 12, pp. 1716-1724, 2002.

[5] A. Tayebi, J. Gomez, J. R. Almagro, I. Gonzalez, and F. Catedra, "Application of EBG structures to the design of a multibeam reflector feed," IEEE Antennas and Propagation Magazine, vol. 56, no. 5, pp. 60-73, 2014.

[6] G. Caille et al., "Electro-magnetic band-gap feed overlapping apertures for multi-beam antennas on communication satellites," in 8th EuCAP, 2014, pp. 963-967.

[7] D. Sanchez-Escuderos, M. Cabedo-Fabres, E. Antonino-Daviu, and M. Ferrando-Bataller, "Extended low-profile planar lens antenna with multilayer metallic-hole array," in 9th EuCAP, 2015, pp. 1-5.

[8] M. Al-Joumayly and N. Behdad, "A generalized method for synthesizing low-profile, band-pass frequency selective surfaces with non-resonant constituting elements," IEEE Transactions on Antennas and Propagation, vol. 58, no. 12, pp. 4033-4041, 2010.

[9] National Instruments, "NI AWR Design Environment," Suite 2014, USA.

[10] M. Al-Joumayly and N. Behdad, "Wideband planar microwave lenses using sub-wavelength spatial phase shifters," IEEE Transactions on Antennas and Propagation, vol. 59, no. 12, pp. 4542-4552, 2011.

[11] D. Sanchez-Escuderos, M. Cabedo-Fabres, E. Antonino-Daviu, and M. Ferrando-Bataller, "Low-profile planar lens with multilevel FSS for directivity enhancement," in IEEE Antennas and Propagation Society International Symposium, 2014, pp. 2076-2077.

[12] C. A. Balanis, Antenna Theory: Analysis and desing. Wiley, 1997. 